\title{
Embedded direct numerical simulation for aeronautical CFD
}

\author{
N. D. Sandham, M. Alam and S. Morin \\ School of Engineering Sciences \\ University of Southampton \\ Southampton, UK
}

\begin{abstract}
A method is proposed by which a direct numerical simulation of the compressible Navier-Stokes equations may be embedded within a more general aeronautical CFD code. The method may be applied to any code which solves the Euler equations or the Favre-averaged Navier-Stokes equations. A formal decomposition of the flowfield is used to derive modified equations for use with direct numerical simulation solvers. Some preliminary applications for model flows with transitional separation bubbles are given.
\end{abstract}

\subsection{INTRODUCTION}

Applied carefully, aeronautical computational fluid dynamics (CFD) codes can deliver useful predictions of flow around aircraft. The methods generally work well when the flow is fully turbulent and remains attached to the surface. The standard methods work much less well when transition to turbulence must be taken into account or when the turbulent flow is subjected to rapid changes in the imposed strain field. The models are particularly challenged in regions where flow separation or reattachment takes place. By contrast direct numerical simulations (DNS), whereby the governing equations are solved in full, are too expensive for complete calculations of flows with aeronautical application, but can deliver accurate solutions of simpler problems without modelling errors. The range of problems that can be tackled by DNS is increasing as the power of computers increases and DNS and the related approach of large-eddy simulation (LES) are being applied to more and more complex geometries and flow fields $(1-3)$. The objective of the current research is to link the two approaches by providing a framework whereby an aeronautical CFD code can provide the context for a detailed DNS, which in turn can automatically feedback improved physical modelling of local phenomena to improve the quality of the overall prediction.

As a model problem we consider the case of a separation bubble, initially laminar, but undergoing transition to turbulence and reattaching as a turbulent boundary layer. In high lift configurations such bubbles may form on the slat and a failure of the flow to reattach ultimately causes stall of the configuration. Prediction of the correct flow with conventional CFD is impossible as typically a transition point must be fixed close to the location where separation takes place, in order to get a converged result. However this fix precludes calculation of the maximum lift coefficient, a quantity forwhich accurate predictions are essential if design is to be optimised.

Several direct simulations of complete separation bubbles have been carried out recently. These simulations include the laminar separation, transition process, turbulent reattachment, and relaxation of the turbulent boundary layer downstream of reattachment. Spalart and Strelets ${ }^{(4)}$ specified a free stream normal velocity profile and simulated a bubble with a length of approximately 300 times the momentum thickness at separation. Alam and Sandham ${ }^{(5)}$ used a similar method for prescribing the free stream velocity distribution but focused on shorter bubbles with lengths of the order of 40 times the momentum thickness at separation. The bubble phenomena reproduced in these simulations closely match earlier experimental work. Besides providing data for understanding flow instability mechanisms (Alam and Sandham), comparisons with aeronautical codes (Spalart and Strelets) and turbulence modelling (Hadzic and Hanjalic(6), Howard et $\left.a^{(7)}\right)$ the simulations also demonstrate the feasibility of simulating flow phenomena at realistic Reynolds numbers on modern computers. It is therefore feasible computationally to consider a direct simulation of a slat separation bubble (which may only occupy $1 \%$ of chord) and a simultaneous CFD simulation of the rest of the flow. The problem which we attempt to address in the present work concerns the details involved in coupling the two approaches. In Section 2.0 we present the governing equation and their Favre-averaged counterparts, such as may be used in CFD codes. In Section 3.0 we introduce a flow decomposition technique using the Euler equations and the Favre-averaged equations as examples. This technique is then applied in Section 5.0, using direct simulation numerical methods given in Section 4.0. Finally in Section 6.0 we present results from a calculation using a Favre-averaged base flow. All the equations and example are presented for compressible flow.

\subsection{GOVERNING EQUATIONS}

\subsection{Instantaneous equations}

The governing equations for mass, momentum and energy conservation may be written in Cartesian tensor notation as: 


$$
\frac{\partial \rho}{\partial t}+\frac{\partial\left(\rho u_{i}\right)}{\partial x_{i}}=0
$$

$$
\frac{\partial\left(\rho u_{i}\right)}{\partial t}+\frac{\partial\left(\rho u_{i} u_{j}\right)}{\partial x_{i}}=-\frac{\partial p}{\partial x_{i}}+\frac{\partial \tau_{i j}}{\partial x_{i}}
$$

$$
\frac{\partial E_{T}}{\partial t}+\frac{\partial\left[\left(E_{T}+p\right) u_{i}\right]}{\partial x_{i}}=-\frac{\partial q_{i}}{\partial x_{i}}+\frac{\partial\left(\tau_{i j} u_{j}\right)}{\partial x_{i}}
$$

where $E_{T}=p\left(e+1 / 2 u_{i} u_{i}\right), e$ being the internal energy. The fluid is assumed to follow the perfect gas law with constant specific heats

$p=(\gamma-1) \rho e$

with $\gamma$ the ratio of specfic heats. Additionally we assume that the fluid is Newtonian and follows Fourier's law for heat conduction:

$\tau_{i j}=\mu\left(\frac{\partial u_{i}}{\partial x_{j}}+\frac{\partial u_{i}}{\partial x_{i}}-\frac{2}{3} \frac{\partial u_{k}}{\partial x_{k}} \delta_{i j}\right)$

$q_{i}=-\kappa \frac{\partial T}{\partial x_{i}}$

where $\mu$ is the viscosity, given by apower law function of temperature, and $\kappa$ is the thermal conductivity. The Euler equations arise when the terms involving viscosity and thermal conductivity are dropped from the right hand sides of Equations (2) and (3).

\subsection{Favre-averaged equations}

In Navier-Stokes calculations with a turbulence model a flow decomposition into an average and a fluctuating component is used. For the compressible equations considered here, a convenient form which preserves the original structure of the equations is the Favre method of mass-weighted variables defined according to

$$
\left.\begin{array}{l}
u_{i}=\widetilde{u}_{i}+u_{i}^{\prime \prime} \\
p=\bar{p}+p^{\prime} \\
T=\widetilde{T}+T^{\prime \prime} \\
E_{T}=\overline{E_{T}}+E_{T}^{\prime} \\
\rho=\bar{\rho}+\rho^{\prime}
\end{array}\right\}
$$

where $\tilde{u}_{i}=\overline{\rho u_{i}} / \bar{\rho}$ and we can also write $E_{T}=\rho E$ with $E=\tilde{E}+E^{\prime \prime}$. It should be noted that for any function $f$ :

$$
\left.\begin{array}{l}
\overline{\rho f^{\prime \prime}}=0 \\
\overline{f^{\prime \prime}}=0
\end{array}\right\}
$$

For low Mach number flows the Favre decomposition reduces to the usual Reynolds-averaged form of the equations.

The Favre-averaged mass conservation equation is

$$
\frac{\partial \bar{\rho}}{\partial t}+\frac{\partial\left(\bar{\rho} \widetilde{u}_{i}\right)}{\partial x_{i}}=0
$$

The momentum equations are: $\frac{\partial\left(\bar{\rho} \tilde{u}_{i}\right)}{\partial t}+\frac{\partial\left(\bar{\rho} \tilde{u}_{i} \tilde{u}_{j}\right)}{\partial x_{i}}=-\frac{\partial \bar{p}}{\partial x_{i}}+\frac{\partial \bar{\tau}_{i j}}{\partial x_{i}}-\frac{\hat{c}\left(\overline{\rho u_{i} u^{\prime \prime}}\right)}{\partial x}$

where

$\bar{\tau}_{\mathrm{ij}}=\bar{\mu}\left(\frac{\partial \widetilde{\mathrm{u}}_{\mathrm{i}}}{\partial x_{\mathrm{j}}}+\frac{\partial \widetilde{\mathrm{u}}_{j}}{\partial x_{\mathrm{i}}}-\frac{2}{3} \frac{\partial \widetilde{\mathrm{u}}_{\mathrm{k}}}{\partial x_{h}} \delta_{i j}\right)$

and we have assumed that

$\overline{\mu\left(\frac{\partial u_{i}^{\prime \prime}}{\partial x_{j}}+\frac{\partial u_{j}^{\prime \prime}}{\partial x_{i}}-\frac{2}{3} \frac{\partial u_{k}^{\prime \prime}}{\partial x_{k}} \delta_{i j}\right)}=0$

The energy equation becomes

$$
\begin{aligned}
& \frac{\partial \overline{E_{T}}}{\partial t}+\frac{\partial\left[\left(\overline{E_{T}}+\bar{p}\right) \widetilde{u}_{i}\right]}{\partial x_{i}}=-\frac{\partial \bar{q}_{i}}{\partial x_{i}}+\frac{\partial\left(\bar{\tau}_{i} \tilde{u}_{i}\right)}{\partial x_{i}} \\
& +\frac{\partial}{\partial x_{i}}\left(\overline{\tau_{i j} u_{j}^{\prime \prime}}-\tilde{u}_{j} \overline{\rho u_{i}^{\prime \prime} u_{i}^{\prime \prime}}-\frac{1}{2} \overline{\rho u_{i}^{\prime \prime} u^{\prime \prime} u^{\prime \prime}}\right)-\vartheta \frac{\left(\overline{\left(\rho e^{\prime \prime} u^{\prime \prime}\right)}\right.}{\partial x}
\end{aligned}
$$

The terms $\overline{\tau_{i j} u_{j}^{\prime \prime}}, \overline{\rho u_{i}^{\prime \prime} u_{j}^{\prime \prime}}, 1 / 2 \overline{\rho u_{i}^{\prime \prime} u_{j}^{\prime \prime} u_{j}^{\prime \prime}}$ and $\overline{\rho e^{\prime \prime} u_{i}^{\prime \prime}}$ can be modelled

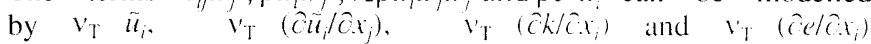
respectively (see Wilcox( ${ }^{(8)}$ ).

\subsection{A DECOMPOSITION APPROACH}

The basic idea of the present contribution is to perform a direct numerical simulation for phenomena such as transition and turbulence using as base flows solutions which may arise from standard aeronautical CFD calculations of compressible flow. A conceptually simple way of arranging this is to consider the total flow to be a superposition of a known base flow and an unknown time-dependent perturbation fromthis to be determined by direct numerical simulation. In the following subsections we derive the relevant equations for a base flow which satisfies either the Euler equations or the Favre-averaged equations for turbulent flow. Similar but simpler equations can be derived for incompressible flow.

\subsection{Euler base flow decomposition}

As a first example of the technique we consider a decomposition into an Euler component $\rho^{I}, u_{i}^{l}, E_{T}^{l}$ which is assumed to have been computed from a separate Euler solver, and a deviation from this which we label $\rho^{D}, u t_{i}^{I}, E_{T}^{l}$. Inserting this decomposition into the governing equations and subtracting the Euler solution of the equations leads to the following equations. For continuity we have:

$\frac{\partial \rho^{D}}{\partial t}+\frac{\partial}{\partial x_{i}}\left(\rho u_{i}^{D}+\rho^{D} u_{i}^{E}\right)=0$

The momentum equation becomes:

$$
\begin{aligned}
& \frac{\partial\left(\rho u_{i}^{D}\right)}{\partial t}+\frac{\partial}{\partial x_{j}}\left[\rho u_{i}^{D}\left(u_{i}^{E}+u_{i}^{D}\right)\right]+ \\
& \left(\rho u_{j}^{D}+\rho^{D} u_{j}^{E}\right) \frac{\partial u_{i}^{E}}{\partial x_{j}}=-\frac{\partial p^{D}}{\partial x_{i}}+\frac{\partial \tau_{i j}^{D}}{\partial x_{j}}+\frac{\partial \tau_{i j}^{E}}{\partial x_{j}}
\end{aligned}
$$


and the energy equation is:

$$
\begin{aligned}
& \frac{\partial E_{T}^{D}}{\partial t}+\frac{\partial}{\partial x_{i}}\left[\left(E_{T}^{D}+p^{D}\right) u_{i}+\left(E_{T}^{E}+p^{E}\right) u_{i}^{D}\right]= \\
& -\frac{\partial q_{i}^{D}}{\partial x_{i}}+\frac{\partial\left(\tau_{i j}^{D} u_{j}\right)}{\partial x_{i}}
\end{aligned}
$$

where

$$
\begin{aligned}
\tau_{i j}^{D} & =\mu\left(\frac{\partial u_{i}^{D}}{\partial x_{j}}+\frac{\partial u_{j}^{D}}{\partial x_{i}}-\frac{2}{3} \frac{\partial u_{k}^{D}}{\partial x_{k}} \delta_{i j}\right) \\
p^{D} & =\left(\rho e^{D}-\rho^{D} e^{E}\right)(\gamma-1) \\
e^{D} & =\frac{1}{\rho}\left[E^{D}-\rho^{D}\left(e^{E}+\frac{u_{i} u_{i}}{2}\right)-\rho^{E}\left(\frac{u_{i}^{D} u_{i}^{D}}{2}+u_{i}^{D} u_{i}^{E}\right)\right]
\end{aligned}
$$

\subsection{Favre base flow decomposition}

A similar procedure leads to equations for the flow-field $\rho^{D}, u_{i}^{D}, E_{T}^{D}$ computed as a perturbation from a Favre-averaged base flow $\rho^{F}, u_{i}^{F}$, $E_{T}^{F}$. For the continuity equation we have:

$$
\frac{\partial \rho^{D}}{\partial t}+\frac{\partial}{\partial x_{i}}\left(\rho u_{i}^{D}+\rho^{D} u_{i}^{F}\right)=0
$$

The momentum equation is:

$$
\begin{aligned}
& \frac{\partial\left(\rho u_{i}^{D}\right)}{\partial t}+\frac{\partial}{\partial x_{j}}\left[\rho u_{i}^{D}\left(u_{j}^{F}+u_{j}^{D}\right)\right]+ \\
& \left(\rho u_{j}^{D}+\rho^{D} u_{j}^{F}\right) \frac{\partial u_{i}^{F}}{\partial x_{j}}=-\frac{\partial p^{D}}{\partial x_{i}}+\frac{\partial \tau_{i j}^{D}}{\partial x_{j}}+\frac{\partial \overline{\left(\rho u_{i}^{\prime \prime} u_{j}^{\prime \prime}\right)}}{\partial x_{j}}
\end{aligned}
$$

and the energy equation becomes:

$$
\begin{aligned}
& \frac{\partial E_{T}^{D}}{\partial t}+\frac{\partial}{\partial x_{j}}\left[\left(E_{T}^{D}+p^{D}\right) u_{i}+\left(E_{T}^{F}+p^{F}\right) u_{i}^{D}\right]= \\
& -\frac{\partial q_{i}^{D}}{\partial x_{i}}+\frac{\partial}{\partial x_{i}}\left(\tau_{i j}^{D} u_{j}+\tau_{i j}^{F} u_{j}^{D}\right) \\
& +\frac{\partial}{\partial x_{i}}\left[\overline{-\tau_{i j} u_{j}^{\prime \prime}}+u_{j} \overline{\rho u_{i}^{\prime \prime} u_{j}^{\prime \prime}}+\frac{1}{2} \overline{\rho u_{i}^{\prime \prime} u_{j}^{\prime \prime} u_{j}^{\prime \prime}}+\overline{\gamma \rho e^{\prime \prime} u_{i}^{\prime \prime}}\right]_{i}^{F}
\end{aligned}
$$

where

$$
\begin{aligned}
& \tau_{i j}^{D}=\mu\left(\frac{\partial u_{i}^{D}}{\partial x_{j}}+\frac{\partial u_{j}^{D}}{\partial x_{i}}-\frac{2}{3} \frac{\partial u_{k}^{D}}{\partial x_{k}} \delta_{i j}\right) \\
& p^{D}=\left(\rho e^{D}-\rho^{D} e^{F}\right)(\gamma-1) \\
& e^{D}=\frac{1}{\rho}\left[E^{D}-\rho^{D}\left(e^{F}+\frac{u_{i} u_{i}}{2}\right)-\rho^{F}\left(\frac{u_{i}^{D} u_{i}^{D}}{2}+u_{i}^{D} u_{i}^{F}\right)\right]
\end{aligned}
$$

\subsection{NUMERICAL METHOD FOR DNS}

The new formulations of the governing equations for mass, momentum and energy given in Section 3.0 are solved numerically using techniques that are suitable for efficient calculation of flows with a wide range of length and time scales. Time discretisation is achieved with an explicit low-storage third-order Runge-Kutta scheme. The time step is fixed to be well within the stability limit to ensure temporal stability. Spatial discretisation is by means of compact Padétype high order schemes with ave-point stencil on the right hand side and a three point stencil on the left hand side. These methods require only a tridiagonal matrix solver. They achieve sixth order accuracy in the inner part of the domain, reducing to third order at boundaries and have good wave resolution properties (Lele $\left.{ }^{(9)}\right)$.

Non-reflecting characteristic boundary conditions are used at the in flow and outflow boundaries. The methods used at the outflow were originally proposed by Thompson (1987) and involve diagonalising the Euler equations and zeroing out the rows containing characteristic velocities that are pointing inwards into the computational domain. At the in flow boundary we use a modification to this approach from Sandhu and Sandham ${ }^{(10)}$ whereby the basic inflow is fixed but with the outgoing characteristic added by integrating it along with the rest of the Navier-Stokes equations. This provides a method of fixing an inflow condition while allowing sound waves to pass smoothly through the inflow boundary. For Navier-Stokes calculations a boundary-layer profile is needed at the inflow. This is found from a similarity solution of the compressible laminar boundary-layer equations.

No slip conditions are used for the lower boundary to simulate a smooth surface. A mixed set of boundary conditions are used at the upper boundary to maintain the flow field. A non-reflecting characteristic boundary condition is used for density and a zero vorticity boundary condition is implemented by setting

$\frac{\partial u^{D}}{\partial y}=\frac{\partial v^{D}}{\partial x}$

and we set

$v^{D}=0$

It should be noted that the boundary conditions specified here need not be the same as those used for the base flow calculation which is one of the strengths of the decomposition method. In the examples in this paper we use Dirichlet conditions for the base flow and mixed Neumann-Dirichlet conditions for the full simulation. Alternative ways of specifying the upper boundary are currently under review to fully utilise the flexibility offered by the decomposition approach.

For all the simulations presented in the following two sections the computational box lengths are $10 \delta$ in the wall-normal direction and $120 \delta^{*}$ in the streamwise direction where $\delta$ represents the displacement thickness of the incoming boundary layer. The grid is stretched in the wall-normal direction to cluster more points in the boundary layer. The number of grid points used were 140 in the wall-normal direction and 100 in the streamwise direction. For viscous calculations the Reynolds number was fixed to be 500 , the Prandtl number to 0.72 , and viscosity varied as $\mu \sim T^{0.67}$.

\subsection{EULER-DNS SIMULATION OF A SEPARATION BUBBLE}

The first step in producing a simulation is to compute an Euler solution. In the anticipated applications this would come from a standard aeronautical Euler solver. Here we consider simpler model problems to demonstrate the techniques. An external velocity profile which can be used to produce a separation bubble is 
$U(x)=A-B \operatorname{Tanh}[C(x-D)]$

where A, B, C and D are constants, which can be fixed to determine the rate and size of the drop in free-stream velocity. For the simulations in this section we use $A=0.91, B=0.09, C=0.08$ and $D=40$, which leads to a free stream drop of $18 \%$ confined within the domain.

We compute the base Euler flow for this simple case by solving the full compressible potential equation

$$
\begin{aligned}
& \frac{\partial^{2} \phi}{\partial x^{2}}+\frac{\partial^{2} \phi}{\partial y^{2}}=\frac{1}{a^{2}}\left[\left(\frac{\partial \phi}{\partial x}\right)^{2} \frac{\partial^{2} \phi}{\partial x^{2}}+\left(\frac{\partial \phi}{\partial y}\right)^{2} \frac{\partial^{2} \phi}{\partial y^{2}}\right] \\
& +\frac{2}{a^{2}} \frac{\partial \phi}{\partial x} \frac{\partial \phi}{\partial y} \frac{\partial^{2} \phi}{\partial x \partial y}
\end{aligned}
$$

where the sound speed $a$ is fixed relative to stagnation conditions $a_{0}$ by

$a^{2}=a_{0}^{2}-\frac{(\gamma-1)}{2}\left[\left(\frac{\partial \phi}{\partial x}\right)^{2}+\left(\frac{\partial \phi}{\partial y}\right)^{2}\right]$

At the subsonic Mach numbers used here the right hand side of Equation (27) is sufficiently small that iterative numerical methods suitable for Laplace's equation still converge. We use a straightforward Gauss-Seidel procedure based on second-order finite differences. The solution is then interpolated onto the actual mesh to be used in the direct numerical simulations.

The simulation using the Euler base flow decomposition from Section 2.1 was run up to time $t=500$. Instantaneous snapshots of the total velocity field (i.e. $u=u^{b}+u^{l}$ ) for $u$ and $v$ are shown on Fig. 1 (a) and (d) together with the streamwise variation of skin friction (b) and the pressure field (c). It can be seen that there is an unsteady separation bubble with weak natural vortex shedding from behind the bubble. A time series of the shedding, as it appears in the streamwise velocity field is shown on Fig. 2. The result that there is vortex shedding for this ow condition is consistent withthe earlier simulation of Rist et $a /(11)$. Additionally time series measurements from within the shedding region ( $\mathrm{Fig}$. 3) show a shedding in the form of wave packets which is also consistent with the previous findings.

(a)

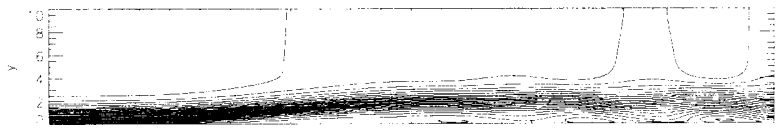

(b)

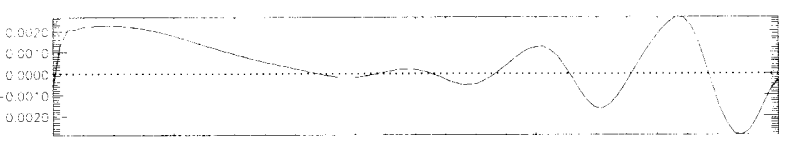

(c)

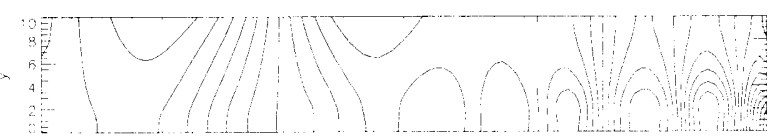

(d)

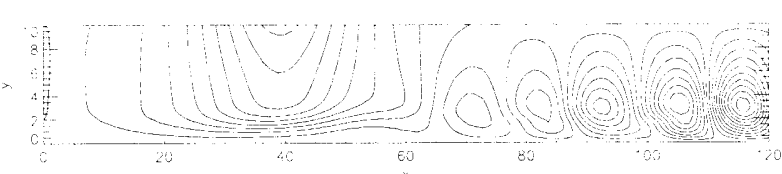

Figure 1. A 2D shedding bubble. Figures top to bottom showing: (a) contours of streamwise velocity, (b) skin-friction, (c) contours of pressure and (d) wall-normal velocity.

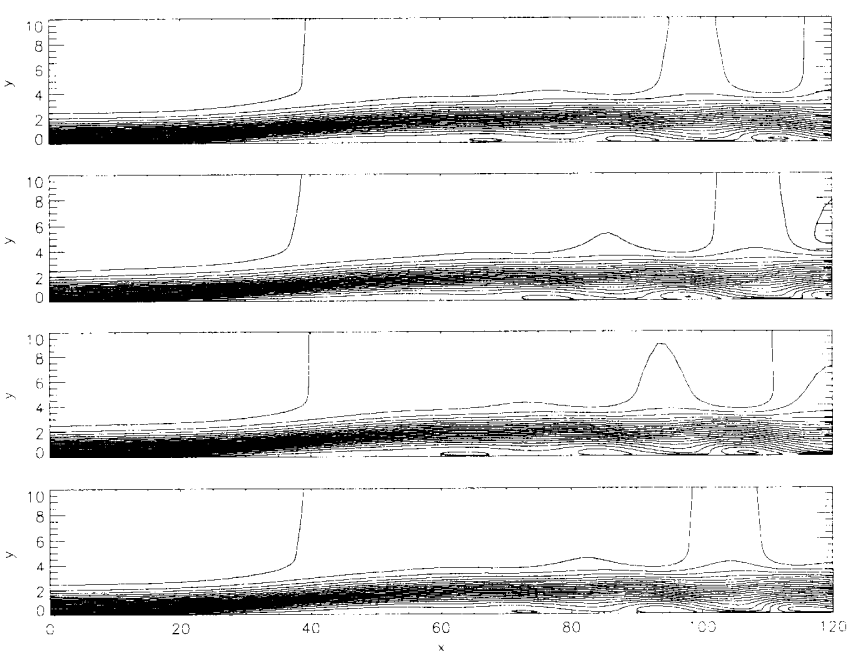

Figure 2. Streamwise velocity contours of a 2D self-excited bubble at different times.

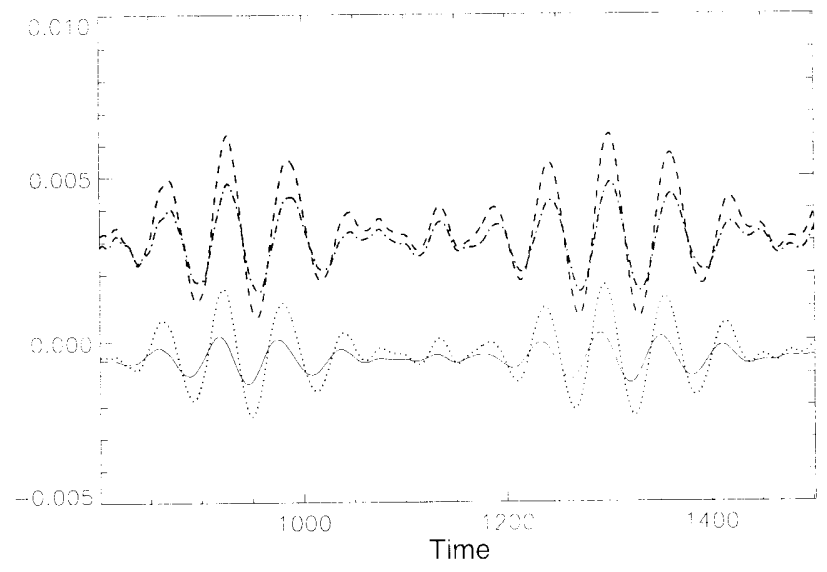

Figure 3. Time history of a wall normal velocity at various heights showing unsteady shedding.

There have been suspicions in the past that shedding maly be triggered numerically so it is useful to have predictions of the same phenomena with computer programs that use different methods. Figure 4 shows the time averaged bubble. Separated flow is found for $50<x<70$.

\subsection{EXAMPLE OF FAVRE-DNS SIMULATION}

For the simulations in this section we use $A=(1.4) . B=0.06$. $C=0.08$ and $D=40$, which leads to a free stream drop of $12 \%$ confined within the domain. This results in a marginally-separated flow and we apply forcing at the inflow to trigger the wres shedding in a deterministic way. The forcing is given by

$u^{\prime}=a_{1} f(y) \operatorname{Sin}(\omega t)$

where $f(y)$ is a normalised funciton of $y$ with a maximum value of unity and a peak at $y=1$ with the constants set as $4=(1) \cdot 01.5$ and $(1)=$ (). 12 .

To generate a Favre base flow we first of all rum an Euler-decomposition calculation for the prescribed potential thil. Results from this calculation are shown on Fig. 5. Again we hat we wex shedding, but this time it is regular and predictable. The rall - from this simulation are then averaged over one cycle of the pertatis acheding to determine the Favre-averaged mean and lluctuation terms. At thispoint we have the statistical equivalent of a calculation of the Favre- 

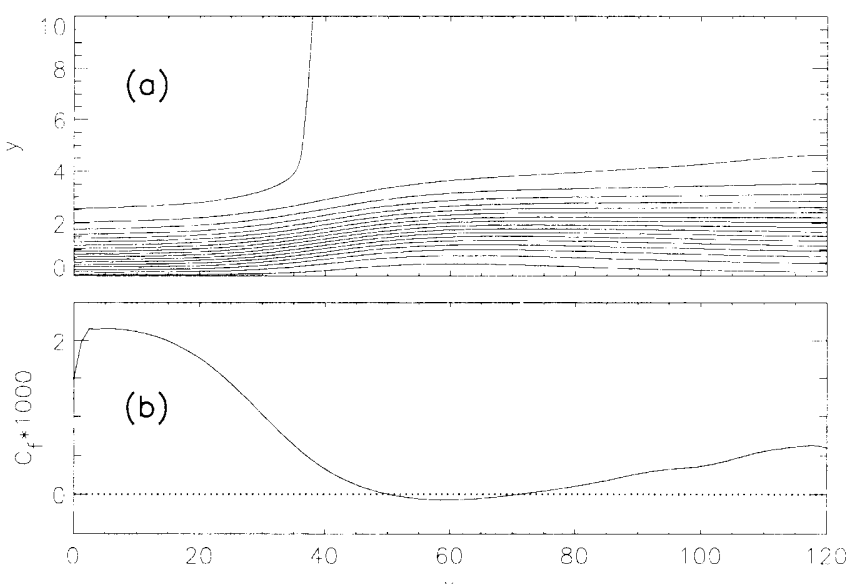

Figure 4. Mean bubble: (a) contours of streamwise velocity and (b) skin-friction.

(a)

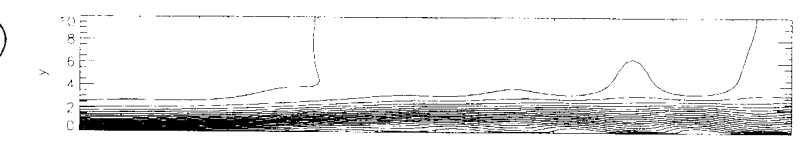

(b)

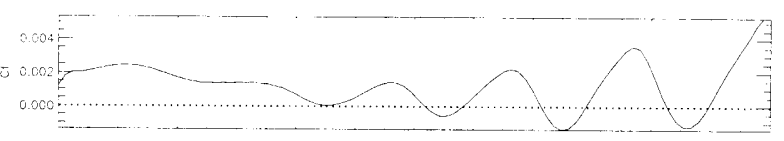

(c)

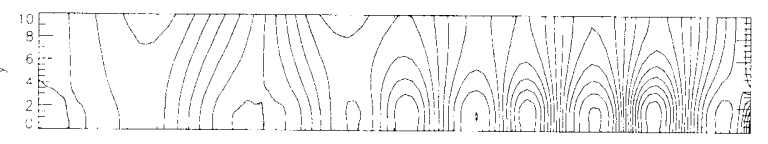

(d)

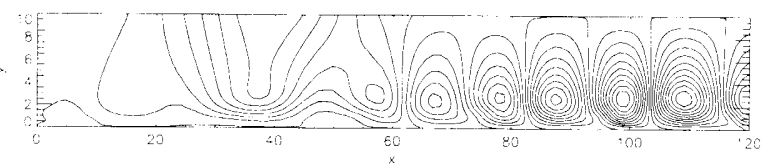

Figure 5. A 2D forced bubble using Euler decomposition. Figures top to bottom showing: (a) contours of streamwise velocity, (b) skin-friction, (c) contours of pressure and (d) wall-normal velocity.

averaged equations using a perfect turbulence model. This is then stored and used as the base flow for a simulation with the

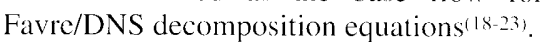

Results from the Favre/DNS simulation are shown on Fig. 6. As expected the flow phenomenon of shedding in response to upstream forcing is reproduced by this method.

Boundary conditions are the main area that requires further work. Ideally boundaries will allow specification of steady inflow profiles, whilst simultaneously allowing waves generated within the simulation to leave smoothly. The present characteristic-based methods lead to a slight drift in the mean flow and we are experimenting with new boundary conditions to overcome this problem.

\subsection{DISCUSSION AND CONCLUSIONS}

We have presented a new decomposition approach to solving acronautical CFD problems. The philosophy of the method is to apply the right methods to the right parts of the flow, using conventional Euler and Favre-averaged-Reynolds-averaged methods for relatively simple parts of the flow, but inserting a time-dependent simulation when the flow phenomena are complex. In the example shown here we consider the case of leading-edge separation bubbles where the idea is for the simulation technique to treat the immediate vicinity of the bubble, feeding back information to the Favre-averaged flow calculation of the rest of the configuration. It is hoped in the future to use the method to predict the overall flow near maximum $C_{l}$ with (a)

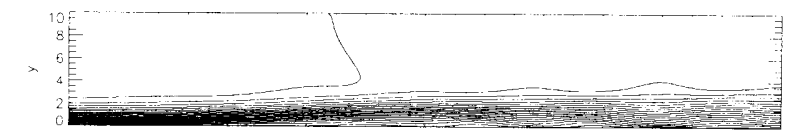

(b)

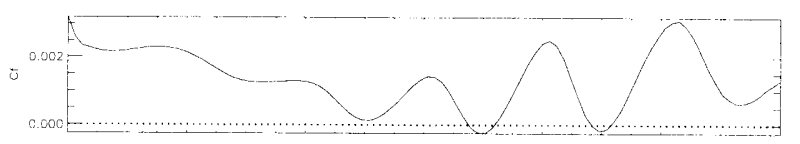

(c)

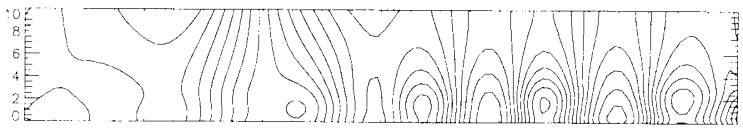

(d)

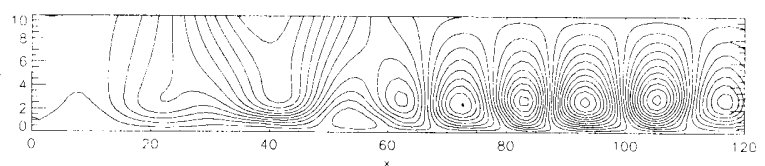

Figure 6. A 2D forced bubble using Favre decomposition. Figures top to bottom showing: (a) contours of streamwise velocity, (b) skin-friction, (c) contours of pressure and (d) wall-normal velocity.

less empiricism than is possible at present. It should be noted that the techniques apply equally well to large-eddy simulation (LES) as to the direct simulation approach used as an example here. Provided the sub-grid models and LES techniques are validated for the complex ow phenomena, this represent a cost-effective way of getting simulation techniques into aeronautical applications.

Another technique which links simulation with conventional modelling approaches is the detached eddy simulation (DES) approach proposed by Spalart ${ }^{121}$. The idea of this approach is to have a simulation technique (LES) which reduces to a turbulence model as the wall is approached. As an example if we have fully separated flow the LES would be used for computing all the vortex events away from the surface, while the conventional one- or two-equation turbulence models would provide reasonable wall boundary conditions for the LES. In many ways this technique is the opposite of that proposed here, where we propose to use DNS/LES near the wall in difficult regions, and use conventional methods elsewhere. Ranges of validity of the two approached remain to be identified in future work.

In principle the techniques given here could be applied to other regions of flow. An example might be to trailing-edge flows, where the feasibility of simulations has already been demonstrated ${ }^{(3)}$. An additional problem here relates to the need to specify turbulent inflow conditions. A simulation approach is often used when accurate in flow data is required,but there is a need for cheap methods of prescribing time-dependent turbulent boundary layer data over a range of Reynolds numbers and upstream strain histories.

\section{ACKNOWLEDGEMENTS}

The authors are grateful for financial support for this project from the Engineering and Physical Sciences Research Council, under grant GR/M 21546. They would also like to acknowledge helpful comments from $\mathrm{Mr}$ Alan Gould of BAE Systems, Sowerby Research Centre.

\section{REFERENCES}

1. Moln, P. and MAHesh. Direct numerical simulation: a tool in turbulence research, Ann Rev Fluid Mechancs, 1998, 30. pp 539-578.

2. AdAMS. N.A. Direct numerical simulation of turbulent compression ramp flow. Theoretical and Computational Fluid Dinamics. 1998, 12 (3). pp 109-129.

3. Yao, Y., Sanibham, N.D., Thomas. T.G. and Will.tams, J.J.R. Study of turbulent trailing-edge flow using direct numerical simulation, Proc Int 
Conf on Turbulence and Shear Flow Phenomena, Santa Barbara, 1999.

4. Spalart, P.R. and Strelets, M.K. Direct and Reynolds-averaged numerical simulation of a transitional separation bubble, $J$ Fluid Mech, 2000, 403, pp 329-349.

5. Alam, M. and Sandham. N.D. Direct numerical simulation of 'short' laminar separation bubbles with turbulent reattachment. $J$ Fluid Mech, 2000, 410, pp 1-28.

6. Hadzic, and Hanjalic, K. Separation-induced transition to turbulence; second-moment closure modelling, $J$ Flow, Turbulence and Combustion, 2000, 63, pp 153-173.

7. Howard, R.J.A., Alam, M. and Sandham, N.D. Two-equation turbulence modelling of a transitional separation bubble, $J$ Flow, Turbulence and Combustion, 2000, 63, pp 175-191.

8. Wil.cox, D.C. Turbulence modelling for CFD, 1994, DCW Industries.

9. LELE, S.K. Compact finite difference schemes with spectral-like resolution, J Computational Physics, 1992, 28, pp 16-42.

10. SAndhu, H.S. and Sandham, N.D. Simulations of leading-edge receptivity to free-stream disturbances, 1996. Internal Report EP-1110 Faculty of Engineering, Queen Mary and Westfield College, University of London.

11. Rist, U. Nonlinear effects of $2 \mathrm{D}$ and $3 \mathrm{D}$ disturbances on laminar separation bubbles, Proc IUTAM Symposium on nonlinear instability of non-parallel flows, New York, 1993.

12. SPALART, P.R. Strategies for turbulence modelling and simulations, 4 th Int Symp Eng Turb Modelling and Measurements, Corsica, May 1999. 